\title{
Comparison of the effect of manual and ventilator hyperinflation on static lung compliance in mechanically ventilated patients with pulmonary acute lung injury/ARDS
}

\begin{abstract}
Purpose: Application of recruitment maneuvers, manual hyperinflation (MHI) and ventilator hyperinflation (VHI) in patients with acute lung injury/adult respiratory distress syndrome (ALI/ARDS) is controversial and concept of more recruitable lung volume in patients with extra-pulmonary ALI/ARDS has also been contradicted. The purpose of the study was to compare effectiveness of $\mathrm{MHI}$ and $\mathrm{VHI}$ in terms of improving static lung compliance $\left(\mathrm{C}_{\mathrm{ST}}\right)$ and oxygenation (pulse oximetric saturation/ fraction of inspired oxygen ratio; $\mathrm{S} / \mathrm{F}$ ratio), altering mean arterial blood pressure(MABP) and heart rate (HR) in patients with pulmonary ALI/ARDS (ALI/ARDSp).
\end{abstract}

Methods: Randomized cross-over study involving 54 mechanically ventilated patients, clinically diagnosed with pulmonary ALI/ARDS

Results: Analysis of variance showed that there was highly significant improvement in $\mathrm{C}_{\mathrm{ST}}$ values with both techniques $(\mathrm{p}<0.01)$, immediately after (Post- 1$), 30$ minutes after (Post-30) and 60 minutes after (Post-60) each intervention. At Post-30 interval, comparing $\mathrm{MHI}$ and VHI using t-test, VHI significantly increased $\mathrm{C}_{\mathrm{ST}}(\mathrm{p}<0.05)$. Comparing $\mathrm{C}_{\mathrm{ST}}$ mean values with critical difference, highest difference was observed between baseline (PRE) and POST-1 time intervals with MHI, and between PRE and POST-30 time intervals with VHI $(p<0.05)$. S/F ratio mean values observed with both VHI and MHI $(p>0.05)$ were statistically non-significant. Also, the two interventions did not show any adverse changes in blood pressure and heart rate.

Conclusion: Hyperinflation as a part of physiotherapy treatment in mechanically ventilated patients with ALI/ARDSp can be performed with equal benefit with both techniques. Patients with ALI/ARDSp also have potential for lung recruitment.
Volume 5 Issue 4 - 2016

\author{
Gitanjali Sikka,' Jeysundar Radhakrishna² \\ 'Assistant Professor, College of Physiotherapy, Pt. B. D. S, \\ University of Health Sciences, Rohtak, Haryana, India \\ ${ }^{2}$ Department of Medicine, University of Alberta, Edmonton, \\ Canada
}

Correspondence: Gitanjali Sikka, Assistant Professor, College of Physiotherapy, Pt. B. D. S, University of Health Sciences, Rohtak, Haryana I2400I, India, Email gitanjali.sikka@gmail.com

Received: August 08, 2016 | Published: August 24, 2016
Abbreviations: ALI, acute lung injury; ARDS, acute respiratory distress syndrome; PEEP, positive end expiratory pressure; $\mathrm{C}_{\mathrm{ST}}$, static lung compliance; $\mathrm{MHI}$, manual hyperinflation; VHI, ventilator hyperinflation; MABP, mean arterial blood pressure; $\mathrm{HR}$, heart rate

\section{Introduction}

Acute lung injury (ALI) and adult respiratory distress syndrome (ARDS) remain major problems in ICU. Despite substantial progress in understanding ARDS pathophysiology, ARDS remains a major clinical problem, and mortality is still as high as $40-46 \% .{ }^{1}$ ARDS is a syndrome of low compliance ${ }^{2}$ and the mainstay of supportive care of ALI/ARDS is mechanical ventilation. Recent developments in the therapeutic approach to ARDS include refinements of mechanical ventilatory support with emphasis on protective lung ventilation using low tidal volumes, increased PEEP with use of recruitment maneuvers to promote reopening of collapsed lung alveoli, prone position as rescue therapy for severe hypoxemia, and high frequency ventilation. Supportive measures in the management of ARDS include attention to fluid balance, restrictive transfusion strategies, and minimization of sedatives and neuromuscular blocking agents. ${ }^{3}$ Data in support of the efficacy of these adjunctive ventilatory therapies remains incomplete, so these modalities are not routinely used.

Several studies recommend the adjunction of recruitment maneuvers (RMs) to mechanical ventilation to limit de-recruitment induced by low tidal volume. ${ }^{5-9}$ Given the uncertain benefit of transient oxygenation improvements in patients with ALI and the lack of information on their clinical outcome effects, the routine use of RMs cannot be recommended or discouraged at this time. RMs should be considered on an individualized basis in patients with ALI with life-threatening hypoxemia. ${ }^{10}$ Additionally, the recruitment maneuvers application has been extensively investigated on extrapulmonary ARDS, on its potential for more recruitable lung volume. But with the end of this myth by studies conducted recently, ${ }^{11,12}$ it is needed to examine RM effecting ALI/ARDSp patients. The use of positive pressure devices has been part of physiotherapy management and currently, the positive pressure has been used in patients in ICU, through the techniques of manual hyperinflation action and, more recently, of ventilator hyperinflation. ${ }^{13-17}$ Ventilator hyperinflation could also result in potential harm to the patient if the ventilator settings were not returned to pre-intervention settings after the procedure. ${ }^{18}$

Studies conducted recently have proved that MHI may be used for clearing secretions, but not accepted in regular practice for treating ARDS. ${ }^{14,16,19-25}$ The effectiveness of MHI and VHI has been compared in intubated and mechanically ventilated patients by many workers ${ }^{14,16,22,26}$ but there is scarcity of literature for studies conducted on patients with ALI/ARDS. Therefore, this study was conducted to investigate and compare the efficacy of MHI and VHI techniques in improving static lung compliance and oxygenation and altering mean 
arterial pressure and heart rate in sedated, paralyzed mechanically ventilated patients with ALI/ARDSp.

\section{Materials and methods}

The study plan was approved from the research ethical committee of Faculty of Applied Sciences, Manav Rachna International University, Faridabad and conducted on patients admitted in ICU's at Paras Hospital, Gurgaon. A total of 54 patients ranging in age from 45 to 70 years, clinically diagnosed with pulmonary ALI/ARDS as per American - European Conference Consensus definition (1994), ${ }^{27}$ mechanically ventilated for less than seven days on volume control mode with PEEP $\leq 10 \mathrm{~cm} \mathrm{H}_{2} \mathrm{O}$, stable hemodynamics with heart rate $=60-100$ beats $/ \mathrm{min} ; \mathrm{MABP}=70-110 \mathrm{~mm} \mathrm{Hg}$ were included in the study. Patients suffering with acute cardiac dysarrthymias/ added cardiac pathology, undrained pneumothorax, obstructive lung disease, haemoptysis of unknown cause, any recent lung transplant, severe bronchospasm, head injuries, chest wall deformity, history of smoking, impaired lung mechanics and conditions where hyperinflation intervention is contraindicated, were excluded from the study.

\section{Procedure}

All the patients who met inclusion criteria were evaluated thoroughly using an evaluation performa and written informed consent from next of kin was obtained. This was a randomized cross over study to control for the effect of day of mechanical ventilation on patient outcome and also to control the severity of lung injury score that may vary from one patient to another. ${ }^{14}$ The order of techniques was randomly selected using computer-generated random permutation. Patients received MHI or VHI in the morning and the alternative intervention in the afternoon, on the same day with a minimum washout interval of three hours. Each treatment session was of 20-25 minutes duration. All patients included in study were sedated with midazolam and morphine on every six hourly basis and paralyzed with pancuronium. Patients were treated in same position for both morning and afternoon sessions, with the affected lung uppermost (bed flat) with unilateral lung disease and in the supine position (bed flat) with bilateral lung compromise. Endotracheal (ET) suctioning was performed approximately 30 minutes before each intervention, so that excess secretions did not influence the dependent variables. Fifteen minutes before the intervention, $\mathrm{FiO}_{2}$ of the ventilator was set as 1.0. After 15 minutes of being ventilated at $\mathrm{FiO}_{2}$ 1.0, a pre intervention (i.e. pre-hyperinflation) measure (PRE) of dependent variables [i.e. static lung compliance and pulse oximetric saturation ratio] was performed. After recording the pre-intervention (baseline) measurements of dependent variables, the intervention (i.e.MHI or VHI) was given. Full aseptic precautions were taken during the procedure, to prevent infection to the patients. Heart rate (HR), electrocardiogram, mean arterial blood pressure (MABP), arterial blood oxygen saturation $(\mathrm{SpO} 2)$ and temperature were monitored using bedside monitor (Philips Intelli Vue MP 40, Philips International B.V., The Netherlands).

\section{Manual hyperinflation}

Manual hyperinflation was performed using Mapleson-"C" circuit and a two liter reservoir bag (Intersurgical Mapleson $\mathrm{C}$ circuit with two liter bag) connected to $100 \%$ wall oxygen at $15 \mathrm{~L}$ per minute. The waveform consisted of an inspiration of three seconds, sustained inspiration for 2 seconds, and a fast release of the valve to ensure a short expiration, during which bag was held compressed. Expiration was passive and unobstructed to facilitate expiratory flow with no PEEP applied. The I: E ratio was 2:1. A manometer (Medisys pressure manometer with $\mathrm{T}$-piece connector) was included in the circuit and patients were manually hyperinflated to a maximum peak airway pressure of $40 \mathrm{~cm} \mathrm{H}_{2} \mathrm{O}$. With a two -handed technique, six sets of six MHI breaths were delivered to the patient. Each MHI set was followed by six tidal breaths to a peak airway pressure of $20 \mathrm{~cm} \mathrm{H}_{2} \mathrm{O}$. The patients were suctioned three times throughout the procedure following every second set of hyperinflation breaths and treatment was of 20 minutes duration.

\section{Ventilator hyperinflation}

Ventilator hyperinflation was given by Siemens Servo 300 A (Siemens-Elema AB, Solna, Sweden) mechanical ventilator by adjusting it to deliver six breaths/min in volume control mode. The tidal volume was increased at the rate of $200 \mathrm{ml}$ increments until a peak airway pressure of $40 \mathrm{~cm}_{2} \mathrm{O}$ was reached, with an inspiratory pause time set at $20 \%$. Then, six mechanical breaths were delivered to the patient. Patients were returned to baseline values and rested for 30 seconds. A total of six sets of six ventilator breaths were delivered to patients following suctioning throughout the procedure on every second set of hyperinflation breaths and treatment lasted for 20 minutes.

\section{Measurements}

Post intervention measures of dependent variables were recorded at one minute after the intervention (POST-1), 30 minutes (POST-30) and 60 minutes (POST-60) after intervention. The formula used for static lung compliance $\left(\mathrm{C}_{\mathrm{ST}}\right)$ was corrected as eVt/Plateau pressurePEEP [28]. Three readings of static pulmonary compliance were taken. S/F ratio act as a surrogate for the oxygenation index. For measuring S/F Ratio, $\mathrm{SpO}_{2}$ was read from monitor and $\mathrm{FiO}$, from the ventilator directly. The average of three readings was taken for $\mathrm{SpO}_{2}$ recording. S/F ratio was calculated using formula $\mathrm{SpO}_{2} / \mathrm{FiO}_{2}$. Heart rate (HR) and Mean arterial blood pressure (MABP) were read directly from monitoring system and recorded before intervention (for MHI intervention recording was done after disconnection from ventilator); 1-minute during intervention; and 1, 5, and 20 minutes following intervention.

\section{Data analysis}

The data collected on different variables at different intervals were subjected to statistical analysis using SPSS software (version 6.0, Chicago, IL).Mean and standard deviation of demographic variables were calculated for all patients. Both the techniques (MHI and VHI) were compared using ' $\mathrm{t}$ '- test for different variables $\left(\mathrm{C}_{\mathrm{ST}}, \mathrm{S} / \mathrm{F}\right.$ ratio, $\mathrm{HR}$ and MABP) at different intervals of time. One way Analysis of Variance was done to test the effect of time interval on different variables and the time interval means were compared using critical difference (C.D.) values $(\mathrm{p}<0.05)$.

\section{Results}

The demographic data of 54 patients, with pneumonia as most common diagnosis, are presented in (Table 1). Analysis of variance showed that there was highly significant improvement in $\mathrm{C}_{\mathrm{ST}}$ values with both MHI $(\mathrm{p}<0.01)$ and VHI $(\mathrm{p}<0.01)$ at POST-1, POST-30, POST-60 time intervals (Table 2). Highest critical difference for the $\mathrm{C}_{\mathrm{ST}}$ mean values was observed between PRE and POST-1 time intervals with MHI, and between PRE and POST-30 time intervals with $\operatorname{VHI}(\mathrm{p}<0.05)$. At POST-30 time interval, VHI showed a significantly higher increase in $\mathrm{C}_{\mathrm{ST}}$ compared to $\mathrm{MHI}(\mathrm{p}<0.05)$ (Table 2, Figure 1). Analysis of variance showed that changes in $\mathrm{S} / \mathrm{F}$ ratio mean values with both VHI $(p>0.05)$ and MHI $(p>0.05)$ were statistically nonsignificant. There was no statistically significant difference between MHI and VHI for mean $\mathrm{S} / \mathrm{F}$ ratio values recorded at various time 
intervals, respectively ( $p>0.05)$ (Table 3 ). Also, the two interventions did not show any adverse changes in blood pressure, heart rate, or heart rhythm.

Table I Subject Characteristics (mean \pm S.D.)

\begin{tabular}{lll}
\hline S. No. & Variables & Mean \pm S.D. \\
\hline I & Age $(\mathrm{yrs})$ & $58.48 \pm I 0 . \mathrm{II}$ \\
2 & Height $(\mathrm{cm})$ & $175.296 \pm 8.62$ \\
3 & Tidal volume $(\mathrm{ml} / \mathrm{Kg})$ & $6.73 \pm 0.44$ \\
4 & PEEP $(\mathrm{cm} \mathrm{H} 2 \mathrm{O})$ & $5.25 \pm 0.7 \mathrm{I}$ \\
5 & FiO2 $(\%)$ & $0.38 \pm 0.06$ \\
\hline
\end{tabular}

S.D, standard deviation

\section{Discussion}

Recruitment maneuver such as hyperinflation have been shown to improve both atelectasis and static pulmonary compliance. ${ }^{13}$ Static lung compliance has also been used as outcome measure to evaluate the efficacy of physiotherapy treatment in previous studies employing use of manual hyperinflation and ventilator hyperinflation as recruitment maneuvers. ${ }^{14-16,20,22,26,29,30}$ The average $C_{\mathrm{ST}}$ value for patients included in our study was $31.53 \pm 5.50 \mathrm{~mL} / \mathrm{cm} \mathrm{H}_{2} \mathrm{O}$, indicating reduction as compared to normal levels. ${ }^{31}$ Improvement in $\mathrm{C}_{\mathrm{ST}}$ at POST-1 time interval with MHI by $13.42 \%$ and with VHI by 12 . $23 \%$ was in accordance to earlier studies ${ }^{14,20}$ and indicated that there may have been a better and greater recruitment of collapsed alveoli immediately after MHI than by VHI. ${ }^{26}$ The application of manual hyperinflation with a larger than normal tidal volume breath coupled with an inspiratory pause adopted in this study, may have facilitated collateral ventilation and effective recruitment of alveoli, thereby improving time-dependent elastic behavior of the lung. There was also a possibility that the manual hyperinflation technique was effective in mobilisation of pulmonary secretions from peripheral to central airways, which were subsequently removed with suctioning, thereby leading to further recruitment of more functional alveolar units. The pathogenesis of pneumonia involves cellular exudates which will likely reduce lung compliance and increase airway resistance, and an improvement in the respiratory mechanics suggesting improved lung function. ${ }^{16,20}$ In addition, the immediate improvement in lung compliance with MHI could be attributed to the use of Mapleson-C circuit which produces significantly larger inspiratory pressures and tidal volumes than other circuits during manual hyperinflation. ${ }^{17,30}$

Barker et al. ${ }^{23}$ reported MHI to be ineffective in improving dynamic pulmonary compliance in mechanically ventilated patients with ALI/ ARDS. The study ${ }^{23}$ had many limitations as study design involved small sample size of heterogeneous group of patients with different murray scores and only a single treatment within a series of potential treatments was investigated. The MHI procedure also had limitations as it was carried out without any pressure manometer and the effect of technique for recruiting injured lung was not investigated. Another study by Paratz et al., ${ }^{24}$ reported significant increase in dynamic pulmonary compliance post-MHI, indicating recruitment of alveoli. The study reports that dynamic lung compliance was sustained at 20 minutes post-MHI $(\mathrm{p}<0.01)$ only in patients with extrapulmonary events considering their potential for more alveolar recruitment This finding is not supported by study methodology as the sample size was small and patient population was of mild to moderate lung disease rather than ALI/ARDS. Also, the physiological basis for explanation of such results has now been contradicted and alveolar recruitment potential has been reported equal in both pulmonary and extrapulmonary forms. ${ }^{11,12,32,33}$ Morrow et al. ${ }^{18}$ also used MHI as recruitment maneuver in ARDS/ALI pediatric patients and reported no increase in dynamic compliance. The study had limitations as participants were not sedated, lack of sufficient power to detect a difference in dynamic compliance and a large number of drop outs. The study reported that recruitment maneuver may have over distended aerated alveolar units before expanding collapsed areas which would cause compliance to either remain unchanged or decrease.

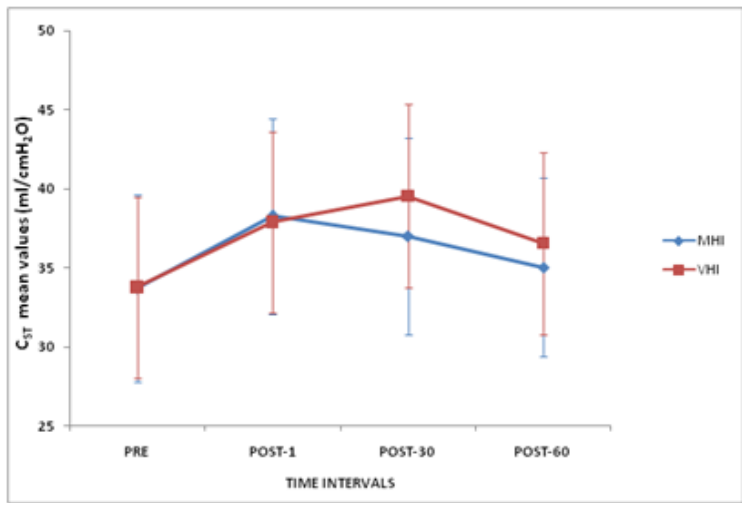

Figure I Changes in $\mathrm{C}_{\mathrm{ST}}$ mean values between manual hyperinflation and ventilator hyperinflation.

$\mathrm{C}_{\mathrm{ST}}$, static lung compliance; $\mathrm{MHI}$, manual hyperinflation; $\mathrm{VHI}$, ventilator hyperinflation; PRE, measurement of dependent variable $\left(\mathrm{C}_{\mathrm{ST}}\right)$ before Intervention; POST-I, measurement of dependent variable $\left(\mathrm{C}_{\mathrm{ST}}\right)$ at I minute after Intervention; POST-30, measurement of dependent variable $\left(\mathrm{C}_{\mathrm{ST}}\right)$ at 30 minutes after Intervention; POST-60, measurement of dependent variable $\left(\mathrm{C}_{\mathrm{ST}}\right)$ at 60 minutes after Intervention.

The improvement in $\mathrm{C}_{\mathrm{ST}}$ at Post-30 time interval with VHI (by17.18\%) was significantly higher than MHI (by 9.63\%) $(\mathrm{P}<0.05)$ in accordance with previous studies. ${ }^{14}$ The decline in compliance levels at POST-30 time interval with MHI can be supported by the de-recruitment in MHI group due to disconnection of the mechanical ventilator, and consequently, the removal of PEEP, and the PEEP availability with VHI group. ${ }^{16,17,26}$ Savian $\mathrm{C}$ et al. ${ }^{16}$ reported both MHI and VHI to be effective in improving static respiratory system compliance over time; however, improvement was only significant for the VHI technique after 30 minutes of treatment. The reason for such results could be that the circuit type used for MHI was a Laerdal manual resuscitation bag, no patient was paralyzed during the study period and study followed a volume-dependent protocol. Ahmed et al. ${ }^{26}$ compared $\mathrm{MHI}$ and $\mathrm{VHI}$ in patients with mitral valve replacement and reported both techniques to be equally effective in improving static lung compliance, but improvements found were statistically nonsignificant at various time intervals $(p<0.05)$. The possible explanation for nonsignificant results with both techniques could be that this study had been conducted on different patient population, based on a volume-dependent hyperinflation protocol and employed use of self-inflating intersurgical 1.5 liter manual resuscitation bag. Dennis et al. ${ }^{22}$ compared MHI and VHI in ventilated intensive care patients and reported that both techniques improved sputum wet weight and dynamic lung compliance but results were statistically non-significant. The possible reason for such results with two techniques could be that patient population included in this study was diverse, ventilated on pressure support ventilation, excess secretions could have influenced dependent variables due to lack of endotracheal suction before either of interventions, MHI was delivered with Laerdal resuscitation bag. The improvement in compliance in the present study at POST-1 and POST -30 time intervals with both the techniques was higher as compared to other studies. ${ }^{14,16,22,26}$ The possible explanation for such results is use of Mapleson C circuit type, patients were sedated and paralyzed, inclusion of homogenous patient population and use of a pressure-dependent hyperinflation protocol. 
Table 2 Comparison of Static lung compliance mean values $\left(\mathrm{C}_{\mathrm{ST}} \mathrm{mL} / \mathrm{cm} \mathrm{H}_{2} \mathrm{O}\right)$ between Manual Hyperinflation and Ventilator Hyperinflation

\begin{tabular}{|c|c|c|c|c|c|c|}
\hline \multirow[b]{2}{*}{ Variable } & \multicolumn{5}{|c|}{ Time Interval (in minutes) } & \multirow[b]{2}{*}{$\begin{array}{l}\text { C.D. (Critical } \\
\text { difference) }\end{array}$} \\
\hline & Intervention & $\begin{array}{l}\text { PRE (before } \\
\text { intervention) }\end{array}$ & $\begin{array}{l}\text { POST-I (I } \\
\text { minute after } \\
\text { intervention) }\end{array}$ & $\begin{array}{l}\text { POST-30 (30 } \\
\text { minutes after } \\
\text { intervention) }\end{array}$ & $\begin{array}{l}\text { POST-60 (60 } \\
\text { minutes after } \\
\text { intervention) }\end{array}$ & \\
\hline \multirow{8}{*}{$\begin{array}{l}\mathrm{CST} \mathrm{mL/cm} \mathrm{H}_{2} \mathrm{O} \\
\text { (mean } \pm \text { S.D.) }\end{array}$} & \multirow{3}{*}{$\begin{array}{l}\text { Manual } \\
\text { Hyperinflation }\end{array}$} & $33.74^{c}$ & $38.27^{a}$ & $36.99^{\mathrm{ab}}$ & $35.03^{b c}$ & \multirow[t]{3}{*}{2.27} \\
\hline & & \pm 5.92 & \pm 6.18 & \pm 6.21 & \pm 5.64 & \\
\hline & & & (13.42\%) & $(9.63 \%)$ & $(3.82 \%)$ & \\
\hline & \multirow{3}{*}{$\begin{array}{l}\text { Ventilator } \\
\text { Hyperinflation }\end{array}$} & $33.75^{c}$ & $37.88^{\mathrm{ab}}$ & $39.55^{a}$ & $36.53^{b}$ & \multirow[t]{5}{*}{2.18} \\
\hline & & \pm 5.72 & \pm 5.7 & $\pm 5.8 \mathrm{I}$ & \pm 5.79 & \\
\hline & & & $(12.23 \%)$ & $(17.18 \%)$ & $(8.23 \%)$ & \\
\hline & t- value & 0.02 & 0.34 & 2.21 & I.37 & \\
\hline & p-value & $0.98^{\mathrm{NS}}$ & $0.73^{\mathrm{NS}}$ & $0.02 *$ & $0.17^{\mathrm{NS}}$ & \\
\hline
\end{tabular}

$\mathrm{C}_{S T}$, static compliance; S.D, standard deviation. Values in parenthesis are mean percentage changes from baseline measurements.

*Measurements are statistically significant at $\mathrm{p}<0.05$

** Highly Significant at $\mathrm{p}<0.01$

*** Means with different superscripts in a column differ significantly $(p<0.05)$. NS: Non-Significant

Table 3 Comparisons of Pulse oximetric saturation ratio (S/F ratio) mean values between Manual hyperinflation and Ventilator hyperinflation

\begin{tabular}{|c|c|c|c|c|c|c|}
\hline \multirow[b]{2}{*}{ Variable } & \multirow[b]{2}{*}{ Intervention } & \multicolumn{4}{|c|}{ Time Interval (in minutes) } & \multirow[b]{2}{*}{$\begin{array}{l}\text { C.D (Critica } \\
\text { difference) }\end{array}$} \\
\hline & & $\begin{array}{l}\text { PRE (before } \\
\text { intervention) }\end{array}$ & $\begin{array}{l}\text { POST- I ( I } \\
\text { minute after } \\
\text { intervention) }\end{array}$ & $\begin{array}{l}\text { POST-30 (30 } \\
\text { minutes after } \\
\text { intervention) }\end{array}$ & $\begin{array}{l}\text { POST-60 (60 } \\
\text { minutes after } \\
\text { intervention) }\end{array}$ & \\
\hline \multirow{8}{*}{$\begin{array}{l}\text { S/F Ratio } \\
\text { (mean } \pm \\
\text { S.D.) }\end{array}$} & & 253.56 & $260.2 a$ & $256.08 a$ & $255.56 a$ & 14.42 \\
\hline & Manual & \pm 38.03 & \pm 38.27 & \pm 38.05 & \pm 37.93 & \\
\hline & & & $(2.6 \%)$ & $(0.99 \%)$ & $(0.79 \%)$ & \\
\hline & & 253.04 & $260.21 \mathrm{a}$ & $260.85 a$ & $257.48 \mathrm{a}$ & 14.34 \\
\hline & $\begin{array}{l}\text { Ventilator } \\
\text { Hyperinflation }\end{array}$ & \pm 38.03 & \pm 37.61 & \pm 37.5 & \pm 38.06 & \\
\hline & 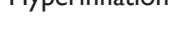 & & $(3.41 \%)$ & $(2.87 \%)$ & $(1.54 \%)$ & \\
\hline & t- value & 0 & 0.28 & 0.65 & 0.34 & \\
\hline & $\mathrm{P}$-value & I.00NS & $0.78 \mathrm{NS}$ & $0.52 \mathrm{NS}$ & $0.73 \mathrm{NS}$ & \\
\hline
\end{tabular}

S/F ratio, pulse oximetric saturation ratio; S.D, standard deviation.

Values in parenthesis are mean percentage changes from baseline measurements. NS: Non-Significant. Means with different superscripts in a column differ significantly $(p<0.05)$

None of the above mentioned studies ${ }^{14,16,22,26}$ comparing MHI and VHI had examined the effects of MHI and VHI on respiratory status of patients at 60 minutes post intervention (POST-60). The present study is the first to evaluate such effects. At POST-60 time interval there was decline in the $\mathrm{C}_{\mathrm{ST}}$ mean values with both the techniques. The improvement in $\mathrm{C}_{\mathrm{ST}}$ with VHI (by $8.23 \%$ ) was higher than MHI (by 3.82\%), but was statistically non-significant. Comparing the results obtained at POST-1 and POST-30 time intervals in the present study, it can be suggested that VHI has better late effects on pulmonary mechanics than MHI, whereas MHI has better immediate effects. ${ }^{16,26}$ However, on comparing POST-1 and POST-60 time interval it can be observed that there is decline in lung compliance improvement obtained with both the techniques. The deterioration in lung compliance at POST-60 time interval with VHI suggests that dependence on the ventilator alone for sustained maintenance of improved lung compliance is not sufficient. VHI can sustain gain in compliance improvement only over a short period of time, as observed in this study at 30 minutes post intervention and has also been supported by previous studies..$^{14,16,26}$ The incompatibility of both $\mathrm{MHI}$ and VHI treatments in sustaining significant improvement in compliance at 60 minutes post treatment can be explained by the loss of recruitment effect. Many studies performed on recruitment maneuvers in ALI/ ARDS patients recommend the use of a recruitment strategy like, HIGH PEEP levels, use of CPAP for a brief period following recruitment maneuver application for promoting their effect for sustained periods..$^{34,35}$ In addition, as the improvement in compliance decreases over time, periodic recruitment is could result in sustained effects.

$\mathrm{S} / \mathrm{F}$ ratio can be used as a surrogate for the $\mathrm{P} / \mathrm{F}$ ratio in the diagnosis of ALI/ARDS. ${ }^{36}$ In the present study, there was an increasing trend in $\mathrm{SpO}_{2} / \mathrm{FiO}_{2}$ ratio with both techniques due to re-expansion of collapsed lung tissues by recruitment maneuver and thereby promoting increase in oxygenation. ${ }^{10}$ The percentage increase in S/F ratio's at various time intervals compared to baseline was higher with VHI, in compliance with other studies reporting MHI not superior to VHI, with respect to arterial oxygenation. ${ }^{14,16,22,26}$ However, the increase in mean S/F ratio values compared to baseline at various time intervals with both techniques could not reach statistical significance. One of the possible reasons for statistically non- significant results for $\mathrm{S} / \mathrm{F}$ ratio could be that, at saturations above $97 \%$ slope of the relationships between $\mathrm{SpO} 2$ and $\mathrm{PaO} 2$ becomes almost zero, and large changes in $\mathrm{PaO} 2$ may result in little or no changes in $\mathrm{SpO} 2 .{ }^{36}$ Also, subsequent fall in percentage indices at various time intervals with both techniques, could be explained by the fact that when oxygenation increases during recruitment maneuver but the improvement is not sustained over time, it is likely that PEEP should be optimized after recruitment maneuver. ${ }^{37}$

Changes in MABP and HR were also compared for MHI and VHI. MABP and heart rate increased in response to either treatment, but returned to same level at 20 minutes post treatment. This implied no significant changes in haemodynamics during either VHI or MHI.VHI 
is equally safe as MHI when applied using the protocol of present study. This finding is in accordance with earlier reports, as there was no variation in haemodynamics along with $\mathrm{MHI}$ and VHI. ${ }^{14,16,22,24,26} \mathrm{As}$ there was no variation in haemodynamics along with MHI and VHI. The possible reason was strict inclusion/exclusion criteria ensuring adequate fluid loading and cardiovascular stability before intervention.

\section{Conclusion}

Both hyperinflation techniques (MHI and VHI) significantly improved static lung compliance. Hyperinflation as a part of physiotherapy treatment in mechanically ventilated patients with pulmonary ALI/ ARDS can be performed with equal benefit using either a manual resuscitation circuit or a ventilator. No adverse changes in hemodynamics were observed during either technique.

\section{Acknowledgments}

None.

\section{Conflicts of interest}

The authors declare there is no conflict of interests.

\section{Funding}

None.

\section{References}

1. Zambon M, Vincent JL. Mortality rates for patients with acute lung injury/ARDS have decreased over time. Chest. 2008;133(5):1120-1127.

2. Bigatello LM, Davignon KR, Stelfox HT. Respiratory Mechanics and Ventilator Waveforms in the Patient with Acute Lung Injury. Respiratory Care. 2005;50(2):235-244.

3. Pierrakos C, Karanikolas M, Scolletta S, et al. Acute Repsiratory Distress syndrome :Pathophysiology and therapeutic options. J Clin Med Res. 2012;4(1):7-16.

4. Levy S. Harrison's Principles of Internal Medicine. 17th edn. USA: The McGraw-Hill Companies, Inc; 2008.

5. Lapinsky S, Mehta S. Bench to bedside review:recruitment and recruiting maneuvers. Critical Care. 2005;9(1):60-65.

6. Constantin JM, Jaber S, Futier E, et al. Respiratory effects of different recruitment maneuvers in acute respiratory distress syndrome. Critical Care. 2008;12(2):1-9.

7. Amato MB, Barbas CS, Medeiros DM. Effect of a protective-ventilation strategy on mortality in the acute respiratory distress syndrome. $N$ Engl J Med. 1998;338(6):347-354.

8. Oczenski W, Hormann C, Keller C, et al. Recruitment maneuvers after a positive end-expiratory pressure trial do not induce sustained effects in early adult respiratory distress syndrome. Anesthesiology. 2004;101(3):620-625.

9. Tugrul S, Akinci O, Ozcan P, et al. Effects of sustained inflation and postinflation positive end-expiratory pressure in acute respiratory distress syndrome:Focusing on pulmonary and extrapulmonary forms. Crit Care Med. 2002;31(3):738-744.

10. Fan E, Wilcox ME, Brower RG, et al. Recruitment maneuvers for acute lung injury:a systematic review. Am J Respir Crit Care Med. 2008;178(11):1156-163.

11. Thille AW, Richard JM, Maggiore SM, et al. Alveolar Recruitment in Pulmonary and Extrapulmonary Acute Respiratory Distress Syndrome. Anesthesiology. 2007;106(2):212-217.

12. Gattinoni L, Caironi P, Cressoni $\mathrm{M}$, et al. Lung recruitment in patients with the acute respiratory distress syndrome. $N$ Engl $J$ Med. 2006;354(17):1775-1786.
13. Scanlan CL, Spearman CB, Sheldon R. Egans's Fundamentals of Respiratory Care. 6th edn. USA: Mosby - Year Book; 1995.

14. Berney S, Denehy L. A comparison of the effects of manual and ventilator hyperinflation on static lung compliance and sputum production in intubated and ventilated intensive care patients. Physiotherapy Research International. 2002;7(2):100-108.

15. Berney S, Denehy L. The effect of physiotherapy treatment on oxygen consumption and haemodinamics in patients who are critically ill. Aust J Physiother. 2003;49(2):99-105.

16. Savian C, Paratz J, Davies A. Comparison of the effectiveness of manual and ventilator hyperinflation at different levels of positive endexpiratory pressure in artificially ventilated and intubated intensive care patients. Heart Lung. 2006;35(5):334-341.

17. Savian C, Chan P, Paratz J. The effect of positive end expiratory pressure level on peak expiratory flow during manual hyperinflation. Anesthesia and Analgesia. 2005;100(4):1112-1116.

18. Morrow B, Futter M, Argent A. A recruitment maneuver performed after endotracheal suction does not increase dynamic compliance in ventilated paediatric patients:a randomized control trial. Aust $J$ Physiother. 2007;53(3):163-169.

19. Maa SH, Hung TJ, Hsu KH, et al. Manual hyperinflation improves alveolar recruitment in difficult-to-wean patients. Chest. 2005;128(4):2714-2721.

20. Choi JS, Jones AY. Effects of manual hyperinflation and suctioning in respiratory mechanics in mechanically ventilated patients with ventilator associated pneumonia. Aust $J$ Physiother. 2005;51(1):25-30.

21. Berney S, Denehy L, Pretto J. Head-down tilt and manual hyperinflation enhance sputum clearance in patients who are intubated and ventilated. Aust J Physiother. 2004;50(1):9-14.

22. Dennis D, Jacob W, Budgeon C. Ventilator versus manual hyperinflation in clearing sputum in ventilated intensive care unit patients. Anaesth Intensive Care. 2012;40(1):142-149.

23. Barker M, Adams S. An evaluation of a single chest physiotherapy treatment on mechanically ventilated patients with acute lung injury. Physiother Res Int. 2002;7(3):157-169.

24. Paratz J, Lipman J, McAuliffe M. Effect of manual hyperinflation on hemodynamics, gas exchange, and respiratory mechanics in ventilated patients. J Intensive Care Med. 2002;17(6):317-324.

25. Paulus F, Binnekade JM, Vroom MB, et al. Benefits and risks of manual hyperinflation in intubated and mechanically ventilated intensive care unit patients:a systematic review. Crit Care. 2012;16(4):R145.

26. Ahmed F, Shafeeq AM, Moiz JA, et al. Comparison of effects of manual versus ventilator hyperinflation on respiratory compliance and arterial blood gases in patients undergoing mitral valve replacement. Heart Lung. 2010;39(5):437-443.

27. Bernard GR, Artigas A, Brigham KL, et al. The American-European Consensus Conference on ARDS:definitions, mechanisms, relevant outcomes, and clinical trial coordination. Am J Respir Crit Care Med. 1994;149(3 Pt 1):818-824.

28. Chang DW. Clinical application of Mechanical Ventilation. 3rd edn. Delmar, USA: Cengage Learning India Private Limited; 2006.

29. Hodgson C, Denehy L, Ntoumenopoulos G, et al. An investigation of the early effects of manual hyperinflation in critically ill patients. Anesthesia and Intensive Care. 2000;28(3):255-226.

30. Hodgson C, Ntoumenopoulos G, Dawson H, et al. The Mapleson C Circuit clears more secretions than the Laerdal circuit during manual hyperinflation in mechanically-ventilated patients:a randomized crossover trial. Aust J Physiother. 2007;53(1):33-38.

31. Nunn JF. Nunnes Applied Respiratory Physiology. 4th edn. Oxford, UK: Butterworth-Heinemann; 1993. 
32. Grasso S, Mascia L, Del Turco M, et al. Effects of recruiting maneuvers in patients with acute respiratory distress syndrome ventilated with protective ventilatory strategy. Anesthesiology. 2002;96(4):795-802.

33. Rouby JJ. Recruitment in pulmonary and extrapulmonary acute respiratory distress syndrome. The end of a myth?. Anesthesiology. 2007;106(2):203-204.

34. Povoa P, Almeida E, Fernandez A, et al. Evaluation of a recruitment maneuver with positive inspiratory pressure and high PEEP in patients with severe ARDS. Acta Anaesthesiol Scand. 2004;48(3):287-293.
35. Medoff BD, Harris RS, Kesselman H, et al. Use of recruitment maneuvers and high- positive end- expiratory pressure in a patient with acute respiratory distress syndrome. Crit Care Med. 2000;28(4):1210 1216.

36. Rice TW, Wheeler AP, Bernard GR, et al. Comparison of the $\mathrm{SpO} 2 /$ FiO2 Ratio and the PaO2/FiO2 Ratio in Patients with acute lung injury or ARDS. Chest. 2007;132(2):410-417.

37. Villagra A, Ochagavia A, Vatua S, et al. Recruitment maneuvers during lung protective ventilation in acute respiratory distress syndrome. $\mathrm{Am} \mathrm{J}$ Respir Crit Care Med. 2002;165(2):165-170. 